\title{
Fibre function and perception during cutaneous nerve block
}

\author{
RODERICK A. MACKENZIE ${ }^{1}$, DAVID BURKE, \\ NEVELL F. SKUSE, AND A. KEITH LETHLEAN \\ From the Unit of Clinical Neurophysiology and The Department of Medicine, \\ Prince Henry Hospital, Little Bay, Sydney, Australia
}

SYNOPSIS In awake human subjects, neural responses in radial nerves to electrical stimulation were recorded with intrafascicular tungsten microelectrodes. Changes in the activity of individual fibre groups during blocking procedures were recorded and correlated with simultaneous alterations in the perception of standardized stimuli. Light touch sensibility in hairy skin appeared to depend on the integrity of A-beta-gamma fibres, cold and pinprick on A-delta fibres, and warmth and dull pain on $\mathrm{C}$ fibres.

The role of individual nerve fibre groups in the appreciation of cutaneous sensation has been the subject of discussion for many years. Conduction of a specific sensory modality by each subgroup of the afferent nerve fibre spectrum has been proposed (Ranson and Billingsley, 1916; Adrian et al., 1931; Heinbecker et al., 1933). Afferent fibres have also been classified according to the method of their excitation as mechanoreceptor, heat, cold, and pain fibres. However, the concept of 'specific' sensory fibres involves consideration of not only the response of a receptor to a specific stimulus, but also the perceptual experience aroused by excitation of the receptor (Hensel, 1952). Monitoring of perception can only be carried out in man, and difficulties in recording volleys in slow fibres in intact man have limited the number of studies in which perception has been correlated with evoked neural activity (Collins et al., 1960; Hensel and Boman, 1960).

Another approach has been to block a cutaneous nerve in a manner which progressively impairs selectively the activity of fibre groups of different sizes, and note changes in perception (Sinclair and Hinshaw, 1950). These correlations have suggested that touch and cold sensation are

\footnotetext{
1 Edwin and Daisy Street Research Fellow in Neurology and Commonwealth Postgraduate Research Scholar.

(Accepted 13 February 1975.)
}

mediated by myelinated fibres and pain and warmth by unmyelinated fibres, but the findings have not been fully accepted because of failure to use standardized thermal stimuli (Sinclair and Glasgow, 1960), as well as uncertainty surrounding the specificity and selectivity of the blocks.

The development of a technique for recording activity from intact nerve fascicles with percutaneous tungsten microelectrodes (Vallbo and Hagbarth, 1968) allows monitoring of the changes which occur during nerve block in awake human subjects. A recent microelectrode study (Torebjörk and Hallin, 1973) has confirmed the selectivity of pressure and local anaesthetic block in man and has correlated changes in the evoked neurogram with changes in the perception of electrical stimuli.

In the present study, this technique was used to correlate changes in the evoked intrafascicular neurogram during selective blocking procedures with changes in the perception of standardized sensory stimuli.

\section{METHODS}

RECORDING TECHNIQUES The examinations were made on the superficial radial nerve of three healthy adult subjects aged 28,30 , and 47 years. None had any evidence of pre-existing neuropathy or other disease. The surface sensory action potential (SAP) was recorded antidromically using standard neuro- 
physiological techniques. To obtain intrafascicular recordings, insulated tungsten microelectrodes with a tip diameter of approximately $1 \mu \mathrm{m}$ were manually inserted percutaneously into the superficial radial nerve at the wrist. The microelectrode was guided into a nerve fascicle by stimulating through the bared tip and adjusting its position until a stimulus of less than $0.5 \mathrm{~V}$ could be perceived. The microelectrode was then used for recording and the amplified signal was monitored on a loudspeaker, displayed on an oscilloscope, and fed into a fixed programme averaging computer (ND 801 Enhancetron 1024) and a tape recorder (Electrodata Model 6300-2).

The fascicular innervation zone was defined by scraping the skin and listening to the evoked neural activity; needle electrodes were then inserted subcutaneously into the area. The averaged evoked neurogram to single electric shocks delivered through the needle electrodes was assessed and on some occasions the position of the microelectrode tip within the fascicle was further adjusted to obtain a satisfactory recording from all fibre groups. Supramaximal stimuli were then applied at 1.0 per second or 1.0 per three seconds when studying fibre groups with a conduction velocity below $2 \mathrm{~m} / \mathrm{s}$. During the time taken for an averaging run, alterations in perception sometimes occurred. If this happened, the results from that block were discarded.

BLOCKING TECHNIQUeS Pressure blocks were produced by screwing a metal plate $1.0 \mathrm{~cm}$ wide across the distal end of the radius, compressing the superficial radial nerve between stimulating and recording sites. The plate did not interfere with circulation in the distal limb. Local anaesthetic blocks were produced at a similar site by subcutaneous injections of lignocaine or procaine. During a single experimental session the nerve under study was initially subjected to one or more local anaesthetic blocks, and after full recovery a pressure block was applied. A period of at least three weeks elapsed between successive experiments on a particular nerve and a limb was not re-used while symptoms persisted.

COMPLICATIONS There were no adverse effects related to the intrafascicular microelectrode penetrations. Recovery from local anaesthetic block was always complete within 30 minutes. On two occasions after pressure block, dysaesthesia in the territory of the compressed nerve lasted several hours, and on one occasion numbness persisted for a week.

SENSORY STIMULI Sensory modalities studied were light touch, pinprick, pain, and thermal sensations (burning heat, warmth, cool, and cold). The subject was prevented from seeing all test stimuli. Pain was tested by delivering single electric shocks to the electrodes inserted in the skin of the fascicular innervation zone. The voltage of the stimulus was adjusted until the shock was perceived as a dull painful jab with an afterpain lasting about one second. All other stimuli were applied to an area of $1.0 \mathrm{~cm}^{2}$ of hairy skin in the fascicular innervation zone which had been selected before the block and marked with Indian ink. Light touch was tested with a wisp of cotton wool, and a moderately sharp pin held manually was used for pinprick sensation. The extremes of thermal sensation were assessed using a lighted match applied close to the skin for heat and an ice cube placed on a small lead plate for cold. Finer temperature discrimination was tested by applying polished copper tubing through which water circulated at predetermined temperatures. The tubing was bent so that the area of contact was approximately $1.0 \mathrm{~cm}^{2}$. Water bath temperature was thermostatically controlled and monitored with mercury thermometers; it was found to vary by less than $0.25^{\circ} \mathrm{C}$. The temperature of the thermal stimulus applied to the skin was taken as the mean of the water bath temperature and that of the returning water, a difference of $0.5^{\circ} \mathrm{C}$. When testing? thermal sensation, each piece of tubing was applied $\frac{1}{2}$ with moderate pressure for five seconds, and theno $\mathbb{D}$ removed. At least 10 seconds elapsed between the application of each thermal stimulus.

Room temperature was maintained at $21^{\circ} \mathrm{C}$. Skin temperature was monitored with a thermocouple and radiant heat was used when necessary to maintain a range of $31-32^{\circ} \mathrm{C}$ throughout the experiment.

\section{RESULTS}

In six experimental sessions the development of nine pressure blocks and one local anaesthetic block was monitored with surface electrode recordings. In a further 10 separate experimental sessions, continuous microneurographic monitoring of 11 pressure blocks and seven local anaesthetic blocks was performed. Changes in sensory modalities were monitored throughout all blocks and the nerve was allowed to recover fully between each phase of an experiment.

PRESSURE BLOCK The earliest change to occur during pressure block was a subjective feeling of dysaesthesia after 20 to 30 minutes in the territory of the nerve being compressed. This was not accompanied by any change in the size or latency of the SAP recorded with surface electrodes, and 

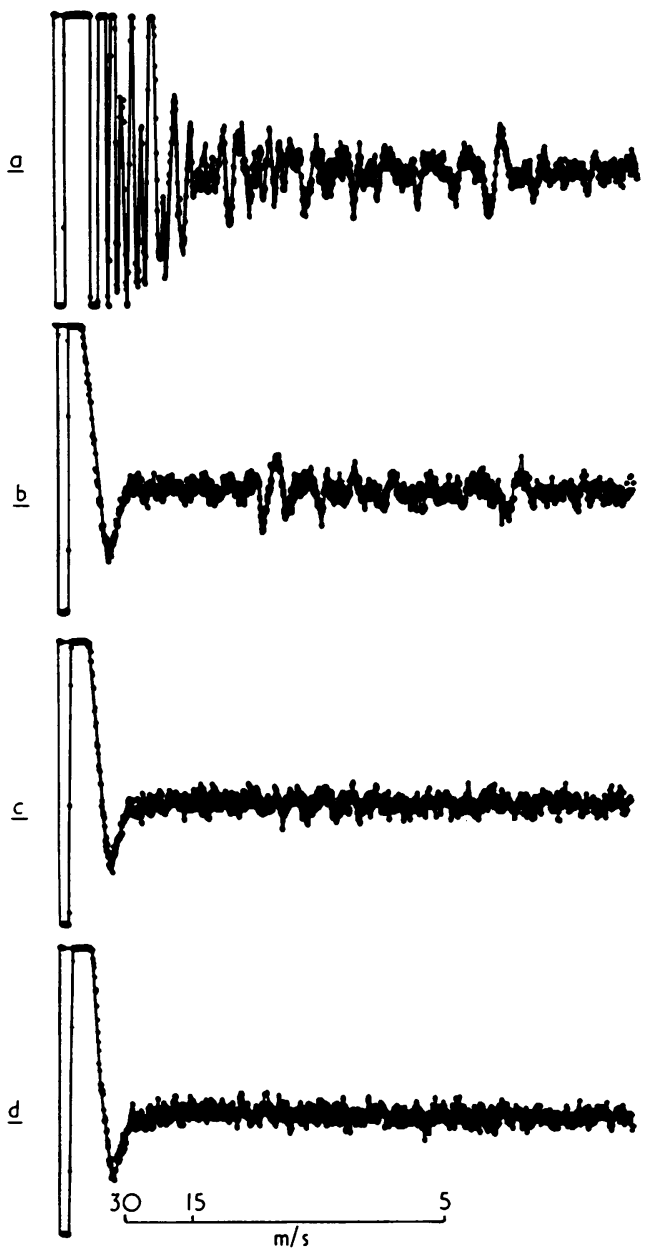

FIG. 1 Changes in myelinated fibre potentials during pressure block. Each diagram consists of the averaged evoked neurogram to 200 supramaximal shocks delivered at 1.0/s. These and the other diagrams have had lines drawn joining points displayed by the averager. a. Normal perception, subjective dysaesthesia. Voltage of A-beta-gamma fibres exceeds input limits of the averager. b. Pressure block at 50 minutes. Light touch absent, pinprick and cold discrimination impaired. Early potentials abolished, late potentials are of lower amplitude and longer latency. c. Pressure block at 55 minutes. Cold and pinprick perception absent. No normal potentials seen. d. Pressure block at 60 minutes. Warmth and pain perception impaired, other sensations absent. No myelinated fibre potentials.

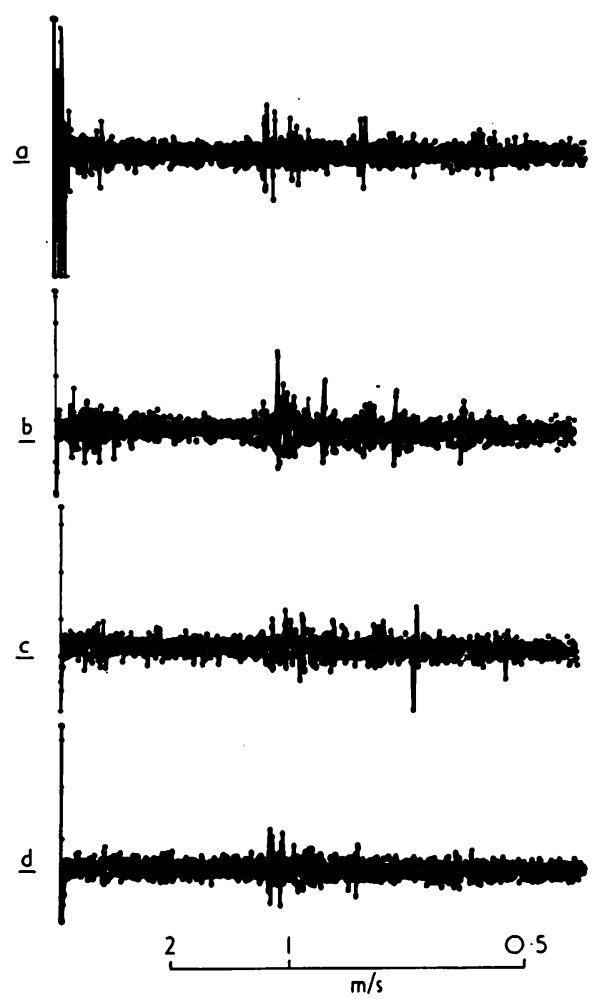

FIG. 2 Changes in unmyelinated fibre potentials during pressure block. Same experiment and similar stages of the block as Fig. 1. Each diagram consists of the averaged evoked neurogram to 50 supramaximal stimuli delivered at 1.0/3.0 s. a. Normal perception. Many potentials averaged, conducting at $0.5-1.2 \mathrm{~m} / \mathrm{s}$. Very early potentials are also seen. b. Fifty minutes. Warmth and pain perception normal. Early potentials absent, $C$ fibre potentials present with increased amplitude. c. Fifty-five minutes. Warmth discrimination impaired. $C$ fibre potentials reduced in amplitude. d. Sixty minutes. Perception of warmth and dull pain further impaired. Reduction in number and amplitude of $C$ fibre potentials.

there was no detectable change in either the averaged intrafascicular neurogram (Fig. 1a) or the audible response from the loudspeaker to skin scraping.

The first objective sensory change was diminished perception of light touch after 30 to 40 minutes of compression. At this stage there was an increase in the latency and diminution in amplitude of the surface SAP evoked by single shocks. The averaged intrafascicular neurogram 
showed an abrupt decrease in the complexity of the potentials conducted faster than $30 \mathrm{~m} / \mathrm{s}$ (A-beta-gamma fibres) and within one to two minutes these potentials had disappeared (Fig. 1b). These changes were accompanied by complete abolition of light touch sensibility from non-hairy areas, although appreciation of light touch in areas of hairy skin involved by the block remained for several more minutes. The loudspeaker response to skin scraping in areas devoid of touch sensation was inaudible, and no SAP could be recorded with surface electrodes.

THERMAL SENSATION Before each block, the temperatures of the 'absolute' thermal stimuli to be applied were adjusted until levels were found which always gave a response of 'warm' or 'cold' by the subject. Average chosen levels for absolute temperature were $27^{\circ} \mathrm{C}$ for cold and $36^{\circ} \mathrm{C}$ for warm. The ability to detect the difference between two successively presented stimuli separated by $1^{\circ} \mathrm{C}$ was also tested, using $27^{\circ}$ and $28^{\circ} \mathrm{C}$ for cold and $35^{\circ} \mathrm{C}$ and $36^{\circ} \mathrm{C}$ for warmth discrimination. These pairs were presented a number of times in randomized fashion and the average of correct, incorrect, and nondiscriminatory responses calculated.

The earliest detectable change in thermal perception during pressure block was an increased number of incorrect responses to discrimination of paired cold stimuli, accompanied by subjective diminution in the intensity of the absolute cold stimulus. In all but three pressure blocks, this change occurred at least five minutes before any change in warmth discrimination. In one of the exceptions, warmth discrimination was impaired one minute before cold, and, in the other two, both were lost together. During the stage of impaired cold discrimination, the averaged intrafascicular neurogram retained fibre potentials of conduction velocity $5-15 \mathrm{~m} / \mathrm{s}$ (A-delta fibres). However, when compared with the preblock recordings, these potentials were of lower amplitude and longer latency (Fig. 1b). Fibre potentials with conduction velocity less than $2 \mathrm{~m} / \mathrm{s}$ (C fibres) were not affected; indeed, $\square_{0}^{\infty}$ during this stage their amplitude was perhaps in- $\frac{\mathrm{D}}{\mathrm{D}} \mathrm{O}$ creased (Fig. 2b) (c.f. Hallin and Torebjörk, 1973).
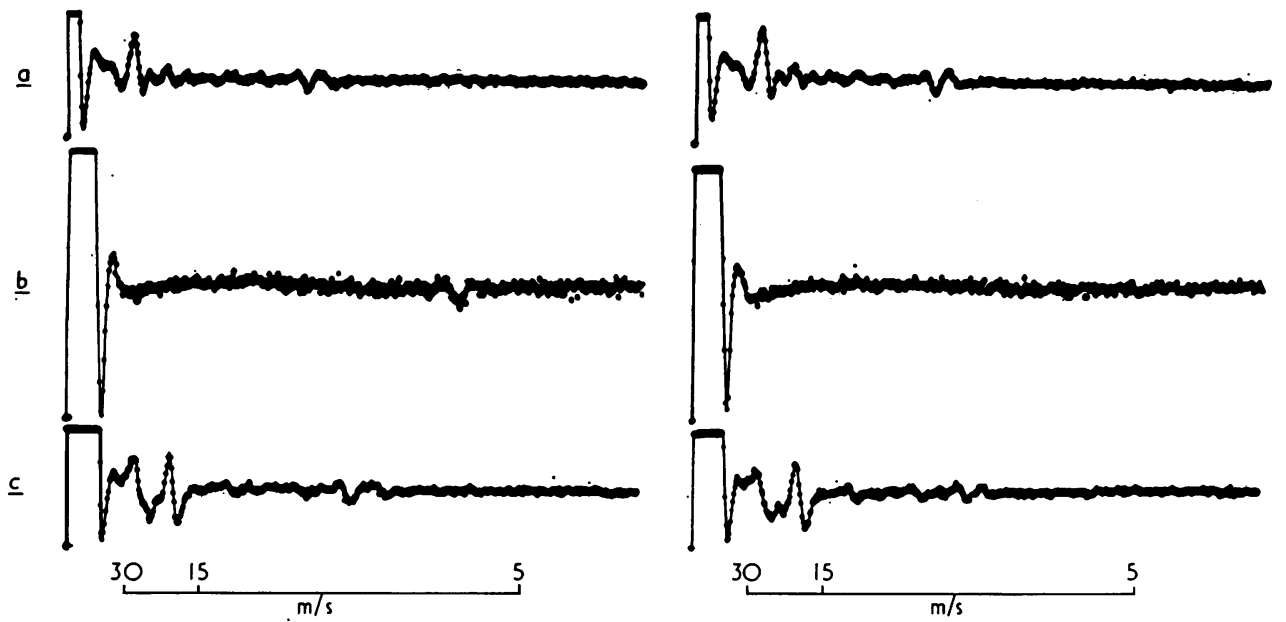

FIG. 3 Pressure block showing persistence of A-delta fibre potential after loss of cold perception. Diagrams on left show averaged response to 100 single shocks at 1.0/s. Diagrams on right show 100 averaged responses to the second of two supramaximal shocks separated by $20 \mathrm{~ms}$ delivered at 1.0/s. a. Pressure block at 30 minutes. Light touch absent, pinprick and cold perception impaired. No difference between the averaged evoked response to single or test stimuli. b. Pressure block at 50 minutes. Cold perception absent. Diagram on left shows delayed A-delta fibre potential; diagram on the right shows no recorded potential to test shock 20 ms after first. c. Pressure block removed. Cold perception normal. No difference between the averaged evoked response to single or paired stimuli. Note: display gain of a was increased to 2.5 times that of $b$ and $c$. 
Progression from impairment of cold discrimination to loss of cold perception took from five to 10 minutes, during which time impairment of warmth discrimination commenced. In all pressure blocks, cold perception was lost when myelinated fibre potentials were completely abolished by pressure (Fig. 1c and d) even though unmyelinated fibre potentials remained (Fig. 2c and d). During this stage, an extreme cold stimulus $\left(6^{\circ} \mathrm{C}\right)$ was felt as an odd 'burning' sensation. When cold perception was absent, A-delta fibre potentials could no longer be seen in the averaged neurogram. A single exception to this general finding is illustrated in Fig. 3. In this instance, an A-delta fibre potential persisted after abolition of perception of all cold stimuli, but was of lower amplitude and longer latency than before. Moreover, during this phase, the response to a second shock $20 \mathrm{~ms}$ later was abolished (Fig. 3b).

When perception of cold was absent and warmth discrimination was impaired, C fibre potentials were still averaged. However, when compared with the neurogram obtained before the block was effective, the $\mathrm{C}$ fibre potentials were reduced in number and their amplitude was less (Fig. 2d).

OTHER SENSATIONS The earliest alteration in pinprick sensation was a diminution in the sharpness of the stimulus. This occurred at the same time as impairment of cold discrimination. As the block progressed, the pin was felt as a dull stimulus only, without any sensation of sharpness. A-delta fibre potentials were no longer recorded at this stage (Fig. 1c) but unmyelinated fibres were still active (Fig. 2c). The first change in the perception of pain during pressure block was a lessening of the jabbing quality of the electrical stimulus, followed by a reduction of the aching interstimulus component. These changes occurred late in the block at the stage when the activity of unmyelinated fibres was reduced (Fig. 2d).

When the block had been in progress for an hour, a spontaneous dull ache began in the territory of the compressed nerve, and continued until the pressure was released. In none of the pressure blocks was unmyelinated fibre activity completely abolished, and some appreciation of pain and heat was always retained.
RECOVERY On removal of the pressure clamp, spontaneous pain disappeared within half a minute and appreciation of the painful electrical stimulus returned to normal. Perception of warmth, cold, and pinprick recovered within a minute, and both $\mathrm{C}$ fibre and A-delta fibre potentials returned within this period. A short period of hypersensitivity to cold and pinprick was noted on some occasions.

Light touch was the last modality to return, accompanied by recovery of the surface SAP evoked by single shocks. Full recovery of touch sensibility was sometimes delayed and the A-betagamma fibre potentials did not regain their former amplitude and complexity until light touch perception was normal. In the case in which symptoms of numbness persisted, recovery of the A-beta-gamma fibre potentials was incomplete, and the surface SAP remained slurred and depressed with prolonged latency for the duration of symptoms.

LOCAL ANAESTHETIC BLOCK Concentrations of lignocaine and procaine of $0.25 \%$ and $0.50 \%$ were found to give quite satisfactory preferential blocks. At higher concentrations, changes occurred too quickly to allow progressive measurements. With dilute solutions, the earliest perceptual change was always a hypersensitivity to cold stimuli which was quite striking and lasted several minutes. No change in $\mathrm{C}$ fibre potentials evoked by single shocks was seen at this early stage. As the block progressed, the first objective sensory change was impairment of warmth discrimination, followed within one or two minutes by reduced appreciation of the dull aching component of the electrical stimulus. The intrafascicular neurogram contained $\mathrm{C}$ fibre potentials of reduced amplitude at this stage, but myelinated fibre potentials were unchanged (Fig. 4).

When further progression of the block occurred, cold discrimination was the next sensation to be affected together with the appreciation of the sharpness of pinprick. Very soon afterwards, heat and pain perception were abolished and intrafascicular recordings showed decrease in A-delta fibre potentials and absence of $\mathrm{C}$ fibre potentials. Light touch was the last modality to be affected. During impairment of light touch perception, the surface SAP was reduced in 

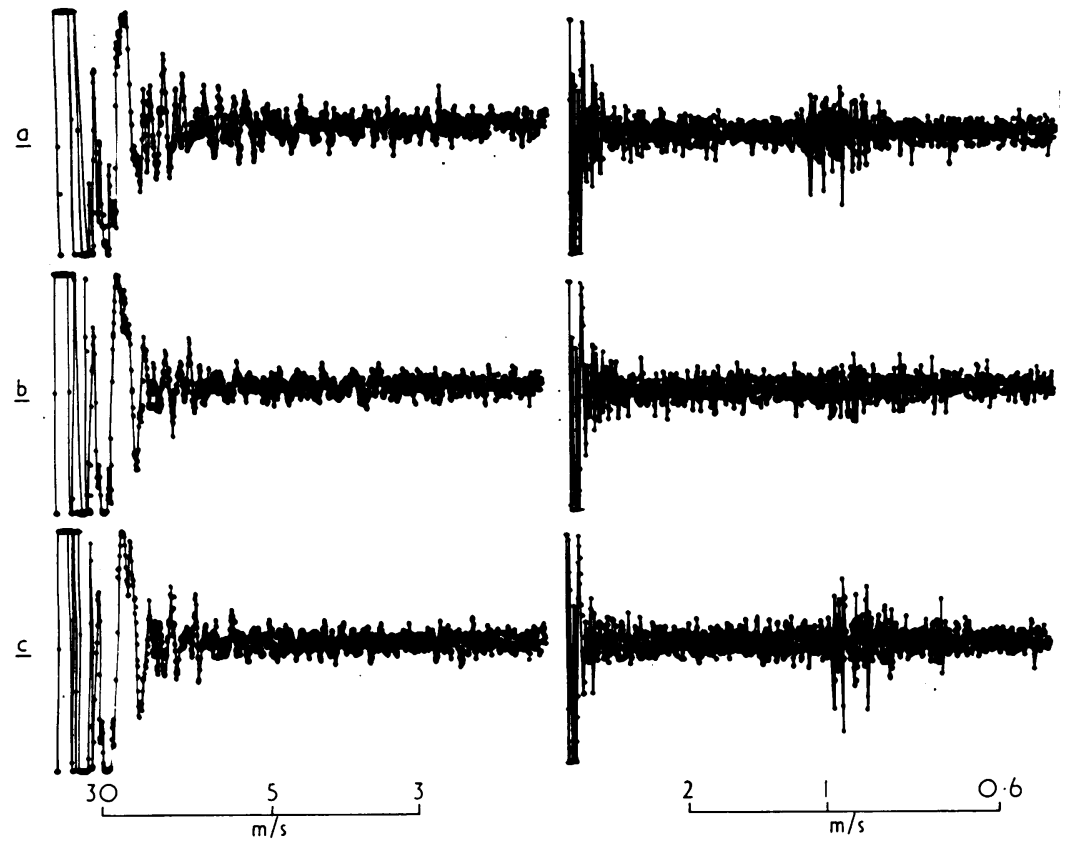

FIG. 4 Changes in myelinated and unmyelinated fibre potentials during block with $0.25 \%$ lignocaine. Diagrams on the left show the averaged evoked neurogram to 100 supramaximal shocks at 1.0/s. Diagrams on the right show the averaged evoked neurogram to 50 supramaximal stimuli at $1.0 / 3 \mathrm{~s}$. Stages of the block are similar in each case. a. Normal perception. b. Fifteen minutes after infiltration. Cold hypersensitivity, impairment of warmth discrimination and pain perception. Myelinated fibre potentials virtually unchanged, $C$ fibre potentials reduced in amplitude. c. Thirty minutes after infiltration. Recovery of perception and fibre potentials (pinprick and touch sensation were unaffected during this block).

amplitude and the latency was prolonged; when light touch sensibility was abolished, the surface SAP was absent and no intrafascicular activity could be evoked with electrical stimulation or skin scraping.

Recovery from local anaesthetic block was slower than for pressure block, but was always complete, with return of surface SAP, intrafascicular neurogram (Fig. 4) and normal perception. There was no clear differentiation of sensory modalities or fibre groups during the process of recovery.

No differences were found in the selectivity of fibre blocks induced by lignocaine and procaine.

\section{DISCUSSION}

Gasser and Erlanger (1929) recorded changes in the evoked action potentials of animal nerves during the production of pressure and local anaesthetic block, and related these changes to alterations in sensation during similar blocking procedures in man. They concluded that the largest fibres were responsible for touch perception, the medium-sized fibres for temperature, and the smallest fibres for pain. Since then, many studies based on animal nerve block experiments have attributed specific sensory functions to particular fibre groups in man (Heinbecker et al., 1933; Landau and Bishop, 1953). However, both the timing of the block of individual fibre groups and the order of involvement of sensory modalities is variable, casting doubts on the validity of such extrapolations. In the present study, these inherent sources of error have been avoided by the simultaneous recording of fibre 
activity and changes in perception. The correlations so determined are independent of both the nature of the block and its rate of development. Further, the range of conduction velocity of fibres associated with each sensory modality can be measured.

The perception of light touch in non-hairy areas was dependent on the function of fibres with conduction velocity greater than $30 \mathrm{~m} / \mathrm{s}$. Touch sensibility in hairy areas was probably also carried by fibres of lower conduction velocity, since its appreciation persisted longer in these areas during pressure block. Similar findings have been reported in cats (Zotterman, 1939; Hunt and McIntyre, 1960) and in primates (Darian-Smith et al., 1973). Psychophysical studies in man suggested that A-beta fibres serve a purely 'tactile' function despite their ability to respond to cold stimuli (Johnson et al., 1973). Further, in disease states involving selective decrease in the number of large myelinated fibres, there is loss of perception of light touch (Dyck and Lambert, 1968).

Dissociation of warmth and cold perception was demonstrated in both pressure and local anaesthetic blocks. The loss of warmth perception many minutes before loss of cold perception in local anaesthetic block has been found by others (Zeng et al., 1973) and the loss of cold before warmth in pressure block is also in agreement with most studies (see Sinclair and Hinshaw, 1950, for review of literature). One study (Sinclair and Glasgow, 1960) found no dissociation of warmth and cold perception during pressure block when limb temperature did not vary. In that study the average temperature of the warm stimulus used was $1{ }^{\circ} \mathrm{C}$ above skin temperature while the average for cold was $4^{\circ} \mathrm{C}$ below skin temperature, biasing the gradient of thermal stimuli to favour the earlier detection of changes in warmth perception. In the present study, both average warm $\left(36^{\circ} \mathrm{C}\right)$ and cold $\left(27^{\circ} \mathrm{C}\right)$ stimuli were $4^{\circ} \mathrm{C}$ from constant skin temperature, stimulus levels found by Cowburn and Fox (1974) in a wide range of subjects to be reliably appreciated as locally warm and cool. These stimuli were lost in a different order in the two blocking procedures, making bias unlikely; furthermore, similar results were obtained by testing the ability to discriminate two thermal stimuli separated by $1{ }^{\circ} \mathrm{C}$, and by the detection of extreme thermal stimuli.

In both pressure and local anaesthetic block, cold perception was impaired contemporaneously with change in conductivity of A-delta fibres and was lost with the disappearance of these potentials. In one experiment, after cold perception was completely abolished, a potential in the A-delta fibre range persisted but its conduction time was considerably increased and its amplitude was reduced. This is consistent with the emergence from the blocked area after a time delay of activity in a reduced number of fibres. However, these remaining fibres would not transmit two volleys $20 \mathrm{~ms}$ apart. The discharge rate at which A-delta fibres in man respond to a cold stimulus is about 50/s (Hensel and Boman, 1960). These partially blocked fibres could not respond at this rate, and it is unlikely that they were capable of conveying reliable information about cold stimuli.

The finding that only the A-delta fibre population in man signals discriminatory cold information to the central nervous system has support from psychophysical studies in man (Johnson et al., 1973) and single fibre analysis in primates (Darian-Smith et al., 1973). These workers concluded that, of the three thermally responsive populations in the median nerve, only the 'cold' fibre population (A-delta) signalled sufficient information to the central nervous system to account for the human subject's capacity to differentiate cooling pulses. A-beta fibres were not sufficiently cold sensitive to play a role in thermal perception, and, although $\mathrm{C}$ fibres had their firing rate suppressed by cold impulses, this was unlikely to account for much of the cold discriminative behaviour of man, since suppression occurred over a very restricted temperature range and was usually complete within a fall of $2^{\circ} \mathrm{C}$.

In the present study, perception of warmth and heat correlated with $\mathrm{C}$ fibre function. This is consistent with the findings in primates (Hensel and Iggo, 1971; Darian-Smith, unpublished data, reported in Darian-Smith et al., 1973) and with recent microelectrode studies in man by other workers (Torebjörk and Hallin, 1972). An interesting observation during pressure block when myelinated fibres were inactive was the interpretation of an extremely cold stimulus as 
'heat'. At this stage, only $\mathrm{C}$ fibres were capable of afferent transmission and presumably central interpretation of the evoked neural response was in terms of the sensory modality normally carried by these fibres.

Perception of the sharp quality of pinprick correlated with the activity of A-delta fibres, and the dull jabbing pain of electrical stimulation with $\mathrm{C}$ fibre activity. In the previous study (Burke et al., 1975), the sharp 'pricking' component of the electrical stimulus became apparent with the excitation of A-delta fibres, and further increases in stimulus intensity caused pricking pain without $\mathrm{C}$ fibre potentials being recorded. The transmission of pain sensation by $\mathrm{C}$ fibres has been inferred from reflex studies in animals (Clark et al., 1935) and has recently been confirmed in man (Torebjörk and Hallin, 1973).

The pin has traditionally been regarded as eliciting two sensations - an early pricking pain abolished by pressure block and a delayed dull pain abolished by local anaesthesia (Lewis and Pochin, 1937). These workers and others (Landau and Bishop, 1953) have suggested that the early response is conducted by A-delta fibres and the later component by $\mathrm{C}$ fibres. However, the experience of the 'second pain' is variable and its existence as a genuine sensory phenomenon has been questioned (Jones, 1956). In view of these doubts and the confirmation of the present studies that pain can be mediated by both $\mathrm{A}$ and $C$ fibres, the role of the pin in neurological diagnosis may be restated. The ability to discriminate a stimulus as 'sharp' or 'blunt' is dependent on A-delta fibre function, while the appreciation of an adequate stimulus as 'painful' can be mediated by A-delta or C fibres. By consideration of both of these components, as well as other sensory modalities, peripheral nerve dysfunctions may be definitively characterized in terms of fibre group involvement.

The authors would like to thank $\mathrm{Mr}$ and Mrs Edwin Street for their wholehearted support of this project. They are also grateful to Dr David Gillies and to Professor James W. Lance for support and criticism throughout the study. Figures were photographed by the Department of Medical Illustration, University of New South Wales. The project was supported by the National Health and Medical Research Council of Australia.

\section{REFERENCES}

Adrian, E. D., Cattell, McK., and Hoagland, H. (1931). Sensory discharges in single cutaneous nerve fibres. Journal of Physiology, 72, 377-391.

Burke, D., Mackenzie, R. A., Skuse, N. S., and Lethlean, A. K. (1975). Cutaneous afferent activity in median and radial nerve fascicles: a microelectrode study. Journal of Neurology, Neurosurgery, and Psychiatry, 38, 855-864.

Clark, D., Hughes, J., and Gasser, H. S. (1935). Afferent function in the group of nerve fibres of slowest conduction velocity. American Journal of Physiology, 114, 69-76.

Collins, W. F., Nulsen, F. E., and Randt, C. T. (1960). Relation of peripheral nerve fibre size and sensation in man. Archives of Neurology, 3, 381-385.

Cowburn, E. J., and Fox, R. H. (1974). A technique for studying thermal perception. Journal of Physiology, 239, 77P-78P.

Darian-Smith, I., Johnson, K. O., and Dykes, R. (1973). 'Cold' fiber population innervating palmar and digital skin of the monkey: responses to cooling pulses. Journal of Neurophysiology, 36, 325-346.

Dyck, P. J., and Lambert, E. H. (1968). Lower motor and primary sensory neurone diseases with peroneal muscular atrophy. 2. Neurologic, genetic and electrophysiological findings in various neuronal degenerations. Archives of Neurology (Chic.), 18, 619-625.

Gasser, H. S., and Erlanger, J. (1929). Role of fiber size in establishment of nerve block by pressure or cocaine. American Journal of Physiology, 88, 581-591.

Heinbecker, P., Bishop, G. H., and O'Leary, J. L. (1933). Pain and touch fibers in peripheral nerves. Archives of Neurology and Psychiatry, 29, 771-789.

Hensel, H. (1952). Physiologie der Thermoreception. Ergebnisse der Physiologie, Biologischen Chemie und Experimentellen Pharmakologie, 47, 166-368.

Hensel, H., and Boman, K. K. A. (1960). Afferent impulses in cutaneous sensory nerves in human subjects. Journal of Neurophysiology, 23, 564-578.

Hensel, H., and Iggo. A. (1971). Analysis of cutaneous warm and cold fibres in primates. Pflüger's Archiv für die gesamte Physiologie, 329, 1-8.

Hunt, C. C., and McIntyre, A. K. (1960). An analysis of fibre diameter and receptor characteristics of myelinated cutaneous afferent fibres in cat. Journal of Physiology, 153, 99-112.

Johnson, K. O., Darian-Smith, I., and La Motte, C. (1973). Peripheral neural determinants of temperature discrimination in man: a correlative study of responses to cooling of skin. Journal of Neurophysiology, 36, 317-370.

Jones, M. H. (1956). Second pain: fact or artifact? Science, $124,442-443$.

Landau, W., and Bishop, G. H. (1953). Pain from dermal, periosteal and fascial endings and from inflammation. Archives of Neurology and Psychiatry (Chic.), 69, 490-504.

Lewis, T., and Pochin, E. E. (1937). The double pain response of the human skin to a single stimulus. Clinical Science, 3 , 67-76.

Ranson, S. W., and Billingsley, P. R. (1916). The conduction of painful afferent impulses in the spinal nerves. American Journal of Physiology, 40, 571-584.

Sinclair, D. C., and Glasgow, E. F. (1960). Dissociation of cold and warm sensibility during compression of the upper limb. Brain, 83, 668-676.

Sinclair, D. C., and Hinshaw, J. R. (1950). Comparison of sensory dissociation produced by procaine and by limb compression. Brain, 73, 480-498. 
Torebjörk, H. E., and Hallin, R. G. (1972). Activity of C fibres correlated to perception in man. In Cervical Pain, pp. 171-178. Edited by C. Hirsch and Y. Zotterman. Pergamon: Oxford.

Torebjörk, H. E., and Hallin, R. G. (1973). Perceptual changes accompanying controlled preferential blocking of $A$ and $C$ fibre responses in intact human skin nerves. Experimental Brain Research, 16, 321-332.

Vallbo, A. B., and Hagbarth, K.-E. (1968). Activity from skin mechanoreceptors recorded percutaneously in awake human subjects. Experimental Neurology, 21, 270-289.

Zeng, M., Fruhstorfer, H., Nolte, H., and Hensel, H. (1973). Dissociated loss of cold and warm sensibility during regional anaesthesia. Pflügers Archiv für die gesamte Physiologie, suppl. 339, R86.

Zotterman, Y. (1939). Touch, pain and tickling: an electrophysiological investigation on cutaneous sensory nerves. Journal of Physiology, 95, 1-28. 